\title{
Dispersion and Property Manipulation of Carbon Nanotubes by Self-Assemibles of Amphiphilic Molecules
}

\author{
Xia Xin, Guiying Xu and Hongguang Li \\ Additional information is available at the end of the chapter \\ http://dx.doi.org/10.5772/51967
}

\section{Introduction}

Over the past two decades, carbon nanotubes (CNTs) have grown as a novel type of nanomaterial and attracted great attention from scientists in different research fields [1-3]. Based on their unique one-dimensional nanostructure, CNTs exhibit excellent mechanical, optical and electronic properties as well as high chemical stability. As claimed by the Nobel laureate Richard Smalley [4], CNTs would be cheap, environmentally friendly, and do wonders for humankind. CNTs were discovered along with the research on fullerenes. In 1991, Iijima from Japan, who at that time was observing fullerenes produced by arc discharge method under high-resolution TEM, observed some tubular structures formed by coaxial cylinders of graphite layers [5]. These structures are nowadays well-known multi-walled carbon nanotubes (MWNTs) with diameters ranging from 2 to several hundred nanometers and lengths of microns. Two years later, single-walled carbon nanotubes (SWNTs) which contain only one cylinder of graphite were also observed and MWNTs can be equally regarded as a group of coaxial SWNTs with different diameters [6]. Theoretically, SWNTs can be regarded as the equivalent by rolling up a single layer of graphite (graphene). The way of the rolling will dominate the diameter and chirality of SWNTs and hence their electronic properties. Taking $a_{1}$ and $a_{2}$ as the basic vector of a graphite layer as shown in Figure 1, the tubes obtained by rolling up this layer along $a_{1}$ are called zigzag tubes, while those along $a_{2}$ are called armchair tubes. The tubes obtained along the vector $a$ will have chirality $\left(a=\mathrm{n} a_{1}+\mathrm{m} a_{2}\right)$. If $\mathrm{n}$ $\mathrm{m}=3 q$ ( $q$ is an integer), the tubes are metallic and can be conductive. When $\mathrm{n}-\mathrm{m} \neq 3 q$, the tubes are semiconducting. 

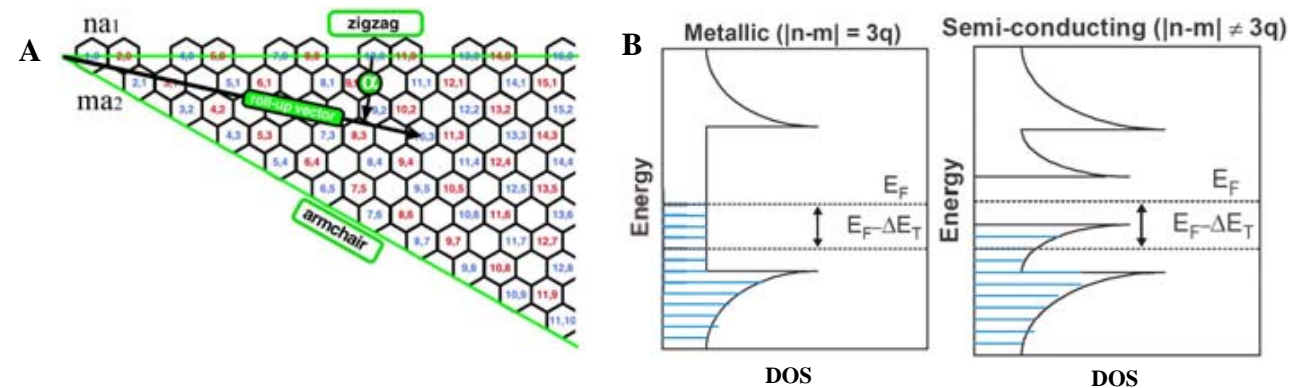

Figure 1. Physical and electronic structures of semiconducting SWNTs. (A) Graphene sheet segment showing indexed lattice points. Nanotubes designated $(n, m)$ are obtained by rolling the sheet from $(0,0)$ to $(n, m)$ along a roll-up vector. The chiral angle a (from 0 to $30^{\circ}$ ) is measured between that vector and the zigzag axis; the tube circumference is the vector's length. (B) The extent of electron transfer is dependent on the density of states in that electron density near $E_{\mathrm{F}}$ leads to higher initial activity for metallic and semimetallic nanotubes $[6,9]$.

This unique geometry at nanometer length scale imparts CNTs many intriguing properties. However, it also leads to a problem which must be solved before many practical applications. That is, the as-produced CNTs, whatever synthetic method is used, are always a mixture of tubes with varying diameter, chirality and length. Besides the continuous effort to directly obtain the desired type of CNTs during synthesis [7], the post-sorting forms an alternative to solve this problem. In the latter case, the CNTs must be well dispersed in a medium. Moreover, the dispersion of CNTs is also a precondition of many fundamental research and practical applications because the aggregation of tubes can significantly lower the promising properties proposed to the single tube of CNTs and bring difficulties to handle these interesting nanomaterials. Unfortunately, in most cases the as-produced CNTs stay as aggregated bundles or ropes instead of single tubes driven by the van der Waals forces and $\pi-\pi$ interactions between adjacent tubes. This aggregation trend becomes more pronounced in the case of SWNTs where the tube has a very high length to width ratio with the order of 100-1000 [8].

From a chemistry viewpoint, an idea to disperse CNTs immediately comes into mind is to covalently link suitable functional groups to the sidewalls or end-caps of the tubes to render the CNTs desired solubility or dispersability in a given solvent. Among various routes to covalently functionalize the CNTs, oxidation using mixed acids [9] has received much interest due to its simplicity and effectiveness. After oxidation, carboxylic groups can be introduced, which opens a route for further functionalizations. This covalent method, however, is proved to disturbing the $\pi$-electrons of the tubes and hence inevitably influencing the intrinsic properties of single tubes. As an alternative and improved solution, in recent years, the so-called noncovalent method using amphiphilic molecules 
to disperse CNTs in aqueous solutions has received much attention [10, 11]. In this chapter, we will first give a brief introduction and overview of the noncovalent method, with an emphasis on SWNTs and recent advances in this field. Then we will focus on the property manipulation of the dispersed tubes in the self-assemblies formed by amphiphilic molecules in water. Typical applications of the dispersed tubes in other research field will also be presented. These include the preparation of functional materials, fabrication of nano-devices and applications in life science. The work related to the dispersion of CNTs in organic solvents using water-insoluble conjugated polymers, which can be also included in the noncovalent method, is out of the scope of this chapter and hence will not be mentioned.

\section{Dispersing CNTs by amphiphilic molecules}

Amphiphilic molecule possesses both hydrophobic and hydrophilic parts in the same molecule [12]. The hydrophobic part is typically the alkyl chain while the hydrophilic part can be an ionized functional group, an ethylene oxide group or the combination of them. Amphiphilic molecule has a special name in colloid and interface science, i.e., surfactant. The surface of CNTs is intrisically hydrophobic and thus has affinity with the hydrophobic part of surfactant. In this case, the hydrophilic part of surfactant stays in water and impedes tube aggregation. This forms the base of using surfactant to disperse and stabilize CNTs. Generally, SWNTs are much more difficult to be dispersed than MWNTs due to the much stronger intertube attractions and thus attracted more attention of researchers in this field. In the following, discussions will mainly be made on SWNTs.

To facilitate the surfactant adsorption onto the tube surface, sonication is needed although example without sonication has also been reported [13]. In a typical dispersion procedure, SWNTs and surfactant are added into water and the mixture is sonicated. Recent report shows that the outermost tubes in a SWNTs bundle are treated more than the innermost tubes and the tubes tend to exfoliate from the bundle ends. Therefore, mechanical exfoliation of the bundles prior to surface treatment must occur in order to obtain individual carbon nanotubes. A mechanism of nanotube isolation from a bundle (Figure 2 i), with the combined assistance of ultrasonication and surfactant adsorption, was proposed [14]. The role of ultrasonic treatment is likely to provide high local shear, particularly to the nanotube bundle end (Figure 2 ii). Once spaces or gaps at the bundle ends are formed, they are propagated by surfactant adsorption (Figure 2 iii), ultimatelym separating the individual nanotubes from the bundle (Figure 2 iv). Since high-power sonication can potentially destroy the tubes, the power of sonication is usually low $(<10 \mathrm{~W})$. On the other hand, to get a good dispersion the sonication time should be sufficiently long (several to tens of hours). After this treatment a SWNTs dispersion with single tubes as well as tube bundles can be obtained. Large tube bundles can be removed from the dispersion by simply gravity sedimentation, while small tube bundles can be stable for weeks. To remove them, ultracentrifugation is recommended. After the sonication-ultracentrifugation circle, SWNTs dispersion up to single tube level can be obtained. 


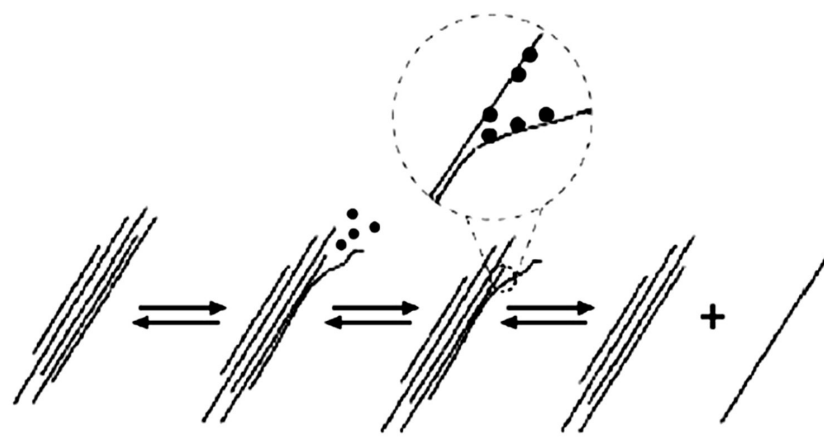

(i)

(ii)

(iii)

(iv)

Figure 2. Mechanism of nanotube isolation from bundle obtained by ultrasonication and surfactant stabilization [14].

\section{Influence of surfactant type}

Up to now, various types of surfactants, both common and uncommon, have been tested to disperse SWNTs in water. Sodium dodecyl sulfate (SDS), which is an anionic surfactant, is among the earliest and most common choices [6, 9, 15], as shown in Figure 3. Later on, investigations have been expanded to cationic, nonionic and zwitterionic surfactants [16-23]. Although both ionic surfactants and nonionic ones can successfully disperse SWNTs in water, the stabilization mechanism for these two categories has subtle difference. While ionic surfactants stabilize the dispersed tubes mainly by electrostatic repulsion, the stabilization mechanism of nonionic surfactant-coated tubes is mainly achieved by steric repulsion. If surfactant molecules are in excess, they can form free micelles. When the size of micelles is above the average distance between the adjacent dispersed tubes, the micelles become difficult to arrange themselves between the tubes and, driven by the depletion attraction, phase separation occurs. Besides the surfactant concentration, the quality of an aqueous dispersion of SWNTs can be also influenced by a variety of other experimental parameters including temperature, $\mathrm{pH}$ and ionic strength.

Amphiphilic macromolecules such as poly (ethylene oxide)-poly (propylene oxide)-poly (ethylene oxide) (PEO-PPO-PEO) tri-block copolymers are also efficient dispersing agents for SWNTs [20, 23-25]. The unique molecular structure and property of these amphiphilic macromolecules lead to some differences in SWNTs dispersion compared to traditional low molecular weight surfactants. For example, the hydrophobic part (PPO segment) is usually longer than that of low molecular weight surfactant and can thus wrap not only on single tubes but also on tube bundles (Figure 4). This will lead to a decreased fraction of single tubes in the dispersion, which seems a disadvantage in cases where single tubes are desired. However, this feature, together with the improved steric repulsion created by the longer hydrophilic part (PEO segment), also enables these amphiphilic macromolecules to disperse and stabilize a large amount 
of tubes into water. This is quite important in applications where the amount of the dispersed tubes is the main concern. Consistent with the larger molecular size, the micelles formed by amphiphilic macromolecules are usually larger compared to those formed by low molecular weight surfactants, which may induce a more pronounced depletion attraction if free micelles are present in the dispersion. Temperature is also an important influencing factor. An increase in temperature can induce desorption of the macromolecule from the tube surface due to a continuous dehydration of the PPO and/or PEO segments.

A
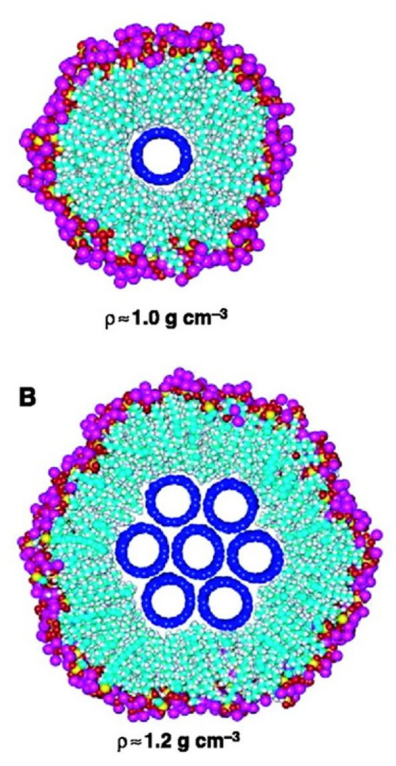
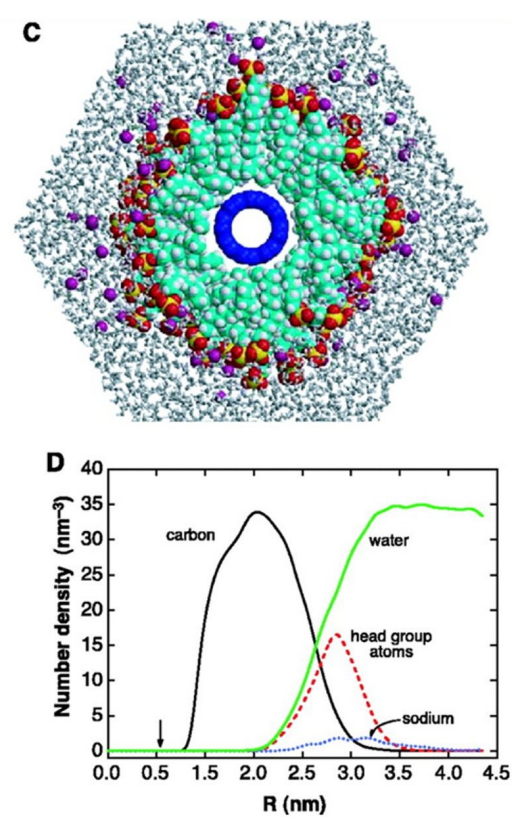

Figure 3. Cross-section model of (A) an individual and (B) a seven-tube bundle embedded in a cylindrical SDS micelle. C) A molecular dynamics simulations of water and the SDS micelle around an individual tube. D) The number density profiles for SDS carbon atoms, sulfate head group atoms, water molecules, and sodium ions [15].

Recent report reveals that the molecular architecture is also an important concern governing the quality of SWNTs dispersion. When the linear PEO-PPO-PEO is branched, an improved capability of SWNTs dispersion and stabilization is observed both experimentally and theoretically. For example, in our laboratory, we have used a starlike amphiphilic block copolymer with PPO-PEO segments (AP432) to disperse CNTs in aqueous solutions. For comparison, two commercially available linear amphiphilic block copolymers, Pluronics L64 and F127, were also selected. It was found that AP432 and F127 can get good CNT dispersions, while L64 was proved to be unable to disperse CNTs. AP432 with five branches could disperse CNTs efficiently at much lower concentrations compared with the linear F127, although it has a smaller molecular weight and shorter terminal EO groups. This indicated 
clearly that, once branched, copolymers would get a much better ability to disperse CNTs $[26,27]$. The detailed information is shown in Figure 5.
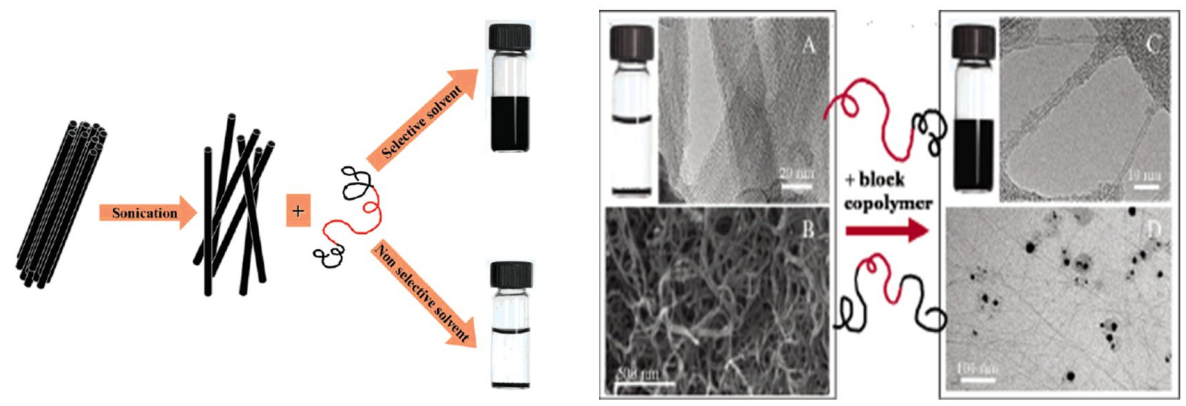

Figure 4. Block copolymers (designated A-B or A-B-A). They are comprised of covalently linked incompatible moieties and disperse SWNT in selective solvents that act as a "good solvent" for one of the blocks (i.e., A), while simultaneously acting as a "poor solvent" for the other block (B). Under these conditions polymer chains may adsorb via physical attachment of the B-block while the A blocks dangle into the solution repelling other polymer-decorated CNT and forming a long-lived dispersion. Nonselective solvents fail to disperse the SWNT [24, 25].

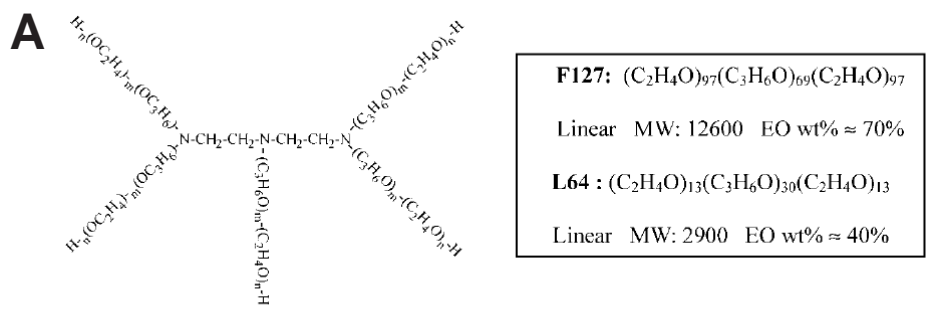

AP432 MW: 7200 EO wt $\% \approx 37.4 \% \mathrm{~m} \approx 15.5, \mathrm{n} \approx 12.2$

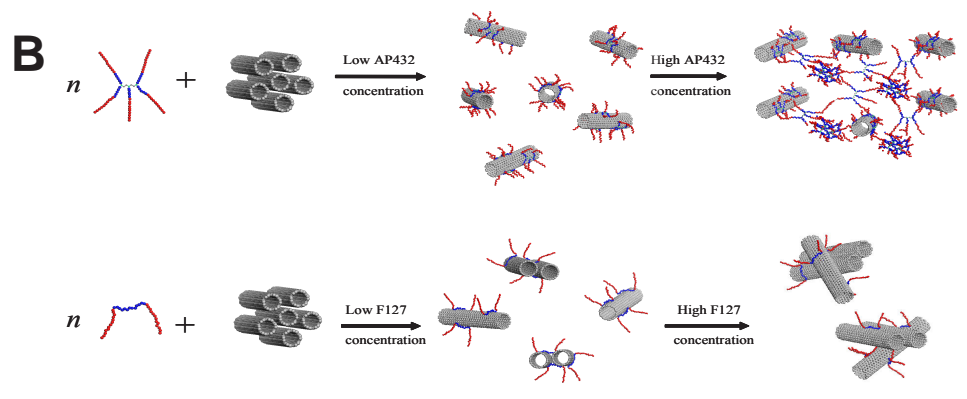

Figure 5. (A) Molecular details of linear (F127 and L64) and star-like amphiphilic block copolymers (AP432). The value of $m$ and $n$ for AP432 is the average value calculated according to the molecular weight and EO content. It is evident that L64 has no ability to disperse CNTs. (B) Schematic Representation of the Possible Mechanism for Nanotube Dispersion by AP432 and F127 [27]. 
Besides the hydrophobic/hydrophobic interaction, $\pi-\pi$ stacking could also play a role in SWNTs dispersion (Figure 6). A typical example is the anionic surfactant sodium dodecylbenzene sulfonate (SDBS) which can greatly enhance SWNTs dispersion in water due to the existance of a benzene ring in the alkyl chain [20,28]. Other examples include the amphiphilic pyrene [29], fluorescein [30], perylene [31] and fullerene [32, 33]. In some cases the affinity between SWNTs and the dispersant can be complicated and not so obvious. There are reports on SWNTs dispersion using polyelectrolytes such as poly (acrylic acid) (PAA) (Figure 7) [34] and naturally occurring macromolecules or biomolecules such as Gum Arabic [35], Hyaluronic Acid [36], starch [37], protein [38] and DNA [39, 40]. The surface activity of these molecules is not as high as traditional surfactants, but satisfactory SWNTs dispersion can be also obtained by optimizing the experimental parameters.

\section{NaDDBS}

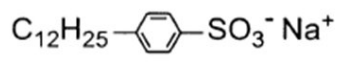

SDS

$$
\mathrm{CH}_{3}\left(\mathrm{CH}_{2}\right)_{11} \mathrm{OSO}_{3}{ }^{-} \mathrm{Na}^{+}
$$

Triton X-100<smiles>[18O]CCO[NH2+]c1ccc([18OH])cc1</smiles>

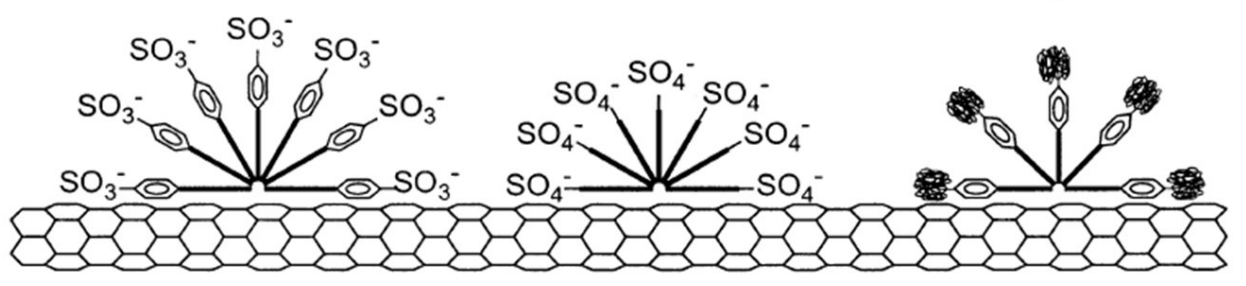

Figure 6. Schematic representation of how surfactants may adsorb onto the nanotube surface. NaDDBS and TX100 were believed to disperse the tubes better than SDS because of their benzene rings. NaDDBS disperses better than TX100 because of its headgroup and slightly longer alkyl chain. The spacing between the benzene rings on the surfactants and the tube surface is large enough to accommodate the $\mathrm{SO}_{3}{ }^{-}$charged groups [28].

A

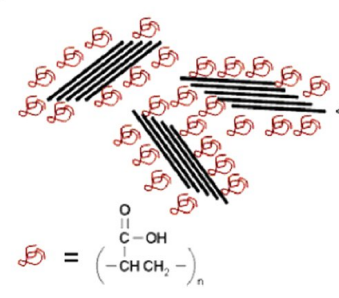

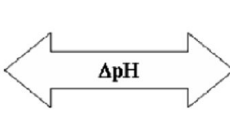

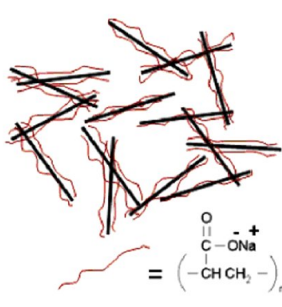

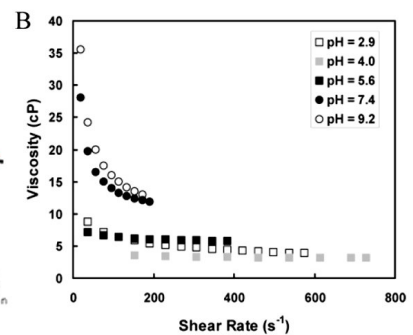

Figure 7. (A) Schematic showing the change in nanotube microstructure that occurs as the pH of poly(acrylic acid) is changed. At low pH, the polymer is uncharged and highly coiled with extensive intrachain hydrogen bonding. At high $\mathrm{pH}$, the carboxylic acid groups are deprotonated and the polymer is more extended as the negatively charged side groups repel one another. (B) Viscosity as a function of shear rate for aqueous suspensions containing 1 wt \% PAASWNT (SWNT is $10 \mathrm{wt} \%$ of the total solids) as $\mathrm{pH}$ is progressively increased [34]. 


\section{Evaluation of the SWNTs dispersion}

The quality of a SWNTs dispersion is evaluated mainly based on the requirement of the specific application. However, there are also some common criterions. The first one is the amount of SWNTs that can be dispersed by surfactants in water. This can be calculated by substracting the undispersed tubes from the total tubes or by simply visual inspections since the dispersion with more tubes usually has a heavier black color. The second and more important criterion is the dispersing extent of the tubes, i.e., bundles or single tubes. This can be checked either directly by imaging methods such as high resolution transmission electron microscopy (HRTEM) or atomic force microscopy observations, or indirectly by spectroscopic characterizations including UV-vis-NIR absorption, Raman spectroscopy and photoluminescence. In the latter case, researchers have shown that once SWNTs are dispersed up to a single tube level, fine structures in both absorption and emission spectra can be observed $[6,9]$. While in a tube bundle, the fine spectroscopic characteristics will be significantly suppressed, highlighting the advantage of SWNTs dispersion by surfactants. The third criterion is the stability of the dispersion. The SWNTs dispersed in water is a kinetically rather than thermodynamically stable system. Thus aggregation and sedimentation can occur with time. If the dispersion is only an intermediated stage in a work and the dispersed tubes will be immediately used for the next step, short period of stability may be sufficient. In some applications, however, long term stability up to weeks or months may be required.

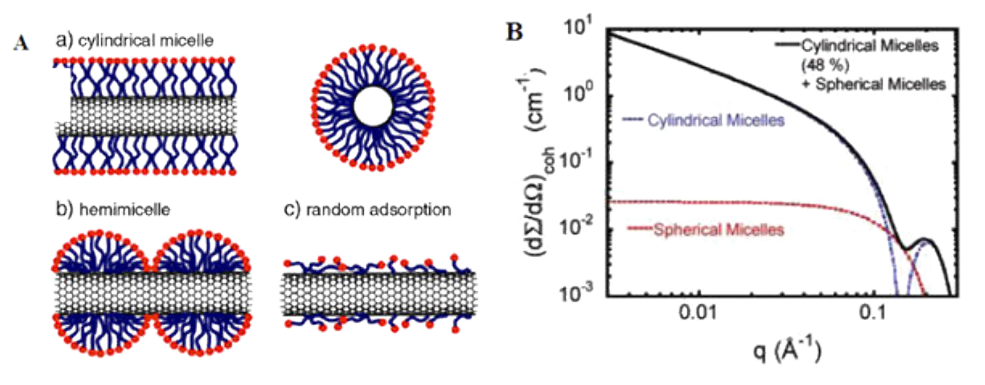

Figure 8. (A) Schematic representations of the mechanism by which surfactants help to disperse SWNT. (a) SWNT encapsulated in a cylindrical surfactant micelle (both cross section and side-view); (b) hemimicellar adsorption of surfactant molecules on a SWNT; (c) random adsorption of surfactant molecules on a SWNT [11]. (B) Coherent SANS intensities calculated for cylindrical (with an embedded nanotube) and spherical core-shell micelles at a concentration of 0.25 wt \% SDS in D2O. The combined cylindrical and spherical micelle prediction shown is calculated on the basis of $48 \%$ of the SDS molecules participating in cylindrical micelles [41].

A concern in SWNTs dispersion is the way of surfactant adsorption on the tube surface. Understanding this can not only help to gain the insight of the interaction mechanism between surfactant molecules and SWNTs, but also provide guidelines to select or design specific surfactants to further improve the quality of the SWNTs dispersions. To fully address this issue experimentally, however, is not an easy task. Up to now, three different adsorption models have been proposed including the cylindrical micelle model [6], semi-sphere model 
[28] and random adsorption model [41]. Figure 8 shows the schematic representations of the mechanism by which surfactants help to disperse SWNT. Although each of them has gained some experimental evidence, the real picture in the microscopic length scale is still to be clarified. In this context computer simulation is frequently adopted and various interaction modes between surfactant molecules and tube surfaces have been proposed [42-44]. Now it is generally accepted that for cylindrical micelle forming surfactant, SWNTs may be encapsulated in the micelles while for spherical micelle forming surfactant, random adsorption may also exist.

\section{SWNTs alighment in ordered surfactant self-assemblies}

In some cases, after the dispersion of SWNTs bundles the tubes are needed to be effectively aligned. SWNTs are anisotropic particles with diameters on the order of nanometers but lengths ranging from micrometers to centimeters. They display most of their expected properties along the tube axis. If the tubes are randomly oriented, the properties will be averaged which should be avoided in some applications such as nanodevice fabrication. The uniform alignment is therefore a crucial condition in these cases. Surfactant can form various self-assemblies in water above the critical micellar concentration $(\mathrm{cmc})$. At medium-to-high concentrations, these self-assemblies can further organize into long range ordered phases called lyotropic liquid crystals (LLC) [45, 46]. An idea immediately comes into mind is that whether these LLC can be utilized to align SWNTs (Figure 9). A typical strategy is that SWNTs are dispersed first in a dilute surfactant aqueous solution before introduced to an LLC matrix since directly disperse SWNTs in a viscous LLC phase could be difficult. The surfactant used for SWNTs dispersion and LLC construction can be the same type or different. The incorporation of the tubes is found not to destroy the LLC matrix. This indirectly proves that the tubes are aligned along the director of the LLC phase since otherwise the system will be energetically unfavorable.

An innovative development in this research field is the fabrication of SWNTs/LLC hybrid by a spontaneous phase separation induced by hydrophilic polymer. In this method, dispersed SWNTs, surfactant and hydrophilic polymer are added into water and homogenized. Initially the concentration of surfactant is well below the critical point for LLC formation and only randomly oriented micelles exist in the solution. If the radius of gyration of the hydrophilic polymer in water is above the average distance between adjacent micelles, the polymer molecules will be driven out and phase separation will occur. The surfactant micelles together with the dispersed SWNTs will be compressed to form a new phase and the hydrophilic polymer forms another. Since the volume shrinks during phase separation, the surfactant concentration in the newly formed phase will be increased to exceed the critical point of LLC formation. This method has been successfully utilized in several surfactant and polymer combinations including nonionic surfactant/nonionic polymer system (Figure 10) [47] and ionic surfactant/polyelectrolyte system where the surfactant and polyelectrolyte have the same sign of charges [48]. The most striking advantage of this method is that the dispersed 
SWNTs take part in the process of LLC formation instead of post-introduction, which is beneficial for tube alignment.
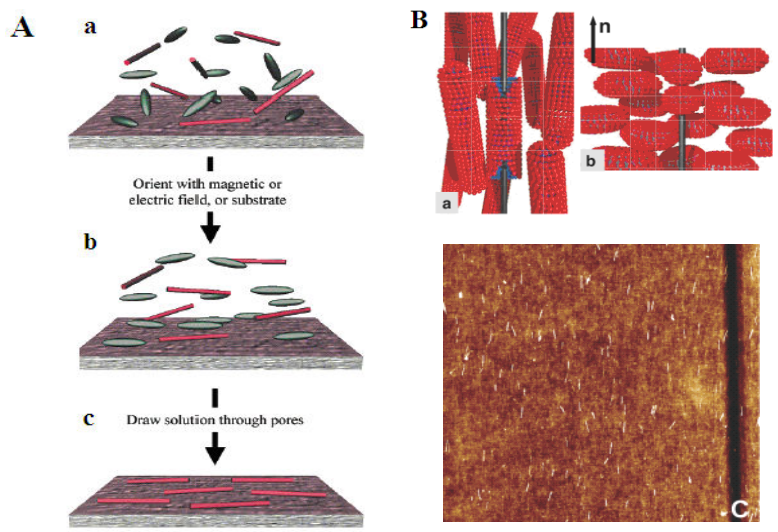

Figure 9. (A) Organizing CNTs with LC solvents. (a) A droplet of liquid crystal (ellipsoids) with suspended carbon nanotubes (rods) is applied to a porous membrane substrate. (b) The bulk LC is then aligned using a grooved surface or external field, which in turn orders the nanotubes. (c) The LC is drained through the porous membrane leaving behind an ordered nanotube film [45]. (B) Schematic illustrations of the mechanism of CNT alignment in a lyotropic nematic LC host, for the cases of rod- and disk-micelle-type adsorption, (a) and (b) respectively, of surfactant molecules on the CNT surface. The CNT is drawn as a vertical black rod in the center of each picture, only partially covered by surfactant molecules for clarity [46]. (C) AFM image of oriented CNT film of MWCNTs from 5CB (50×50 $\mu \mathrm{m})$ [45].

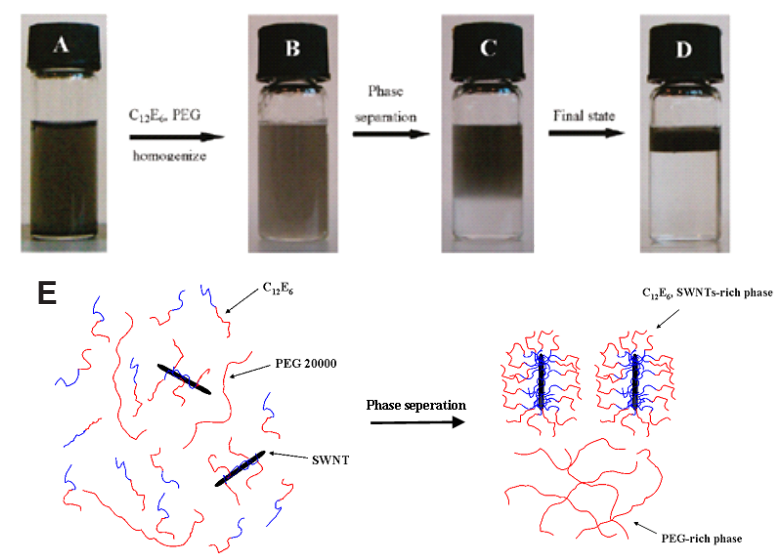

Figure 10. Schematic illustration of the phase separation process. (A) SWNTs dispersion in 0.1 wt $\% \mathrm{C}_{12} \mathrm{E}_{6}$ aqueous solution; (B) mixture of SWNTs dispersion, $\mathrm{C}_{12} \mathrm{E}_{6}(10 \mathrm{wt} \%)$ and PEG(20 wt \%) after being homogenized; (C) intermediate stage during phase separation; (D) final state after equilibrium. (E) Schematic Representation of the Phase Separation Process in the Four Component Mixture of the Surfactant $C_{12} E_{6}$, PEG 20000, SWNTs, and Water [47]. 
The dispersed SWNTs could also be aligned in randomly oriented surfactant micelles. For example, Tannenbaum's group probed the effects of shear flow on the alignment of dispersed single-walled carbon nanotubes in polymer solutions. Two different systems were compared: Single-walled carbon nanotubes dispersed using an anionic surfactant (sodium dodecyl benzene sulfonate, NaDDBS) and single-walled carbon nanotubes dispersed using an anionic surfactant and a weakly binding polymer (carboxyl methylcellulose, CMC). In this case, an additional force, typically shear, will be needed. It was determined that the addition of a weakly binding polymer serves two purposes: constituting the polymer matrix in which the SWNT will be dispersed and aligned and providing a secondary mechanism for the promotion of carbon nanotube dispersion [49]. The results showed that the tubes are found to be preferentially align along the direction of shear flow, as shown in Figure 11. Some reports also revealed that the presence of SWNTs can have a pronounced effect on the ordering of micelles [50]. This disadvantage of such method is, however, once the shear flow is stopped, the tubes and micelles tend to be disordered again.
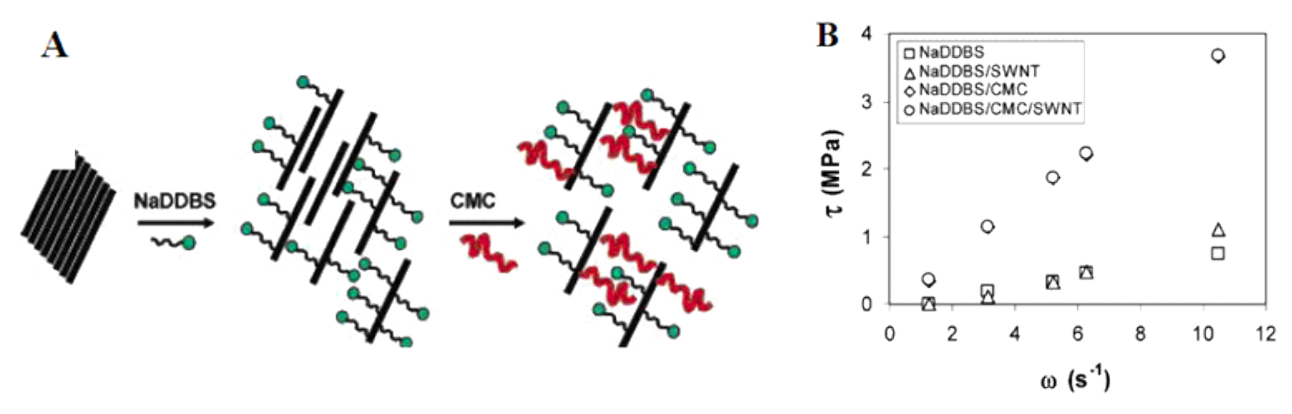

Figure 11. (A) Schematic representation of the stabilization mechanism of carbon nanotubes: Interaction between SWNT with NaDDBS, followed by the addition of CMC. It is important to note that the molecules were not drawn to scale, and the dimension of the polymer molecules is about 2 orders of magnitude larger that the dimension of the surfactant molecules $(R g \approx 1000 \AA$ for CMC as compared to $\approx 20 \AA$ for NaDDBS); (B) Summary of the calculated shear stresses of the various SWNT-containing solutions: Plots of shear stresses as a function of angular velocity [49].

\section{Applications of the surfactant-coated SWNTs}

The formation of well-dispersed SWNTs in surfactant aqueous solutions opens the door for further investigation and practical applications of these interesting nanomaterials, as shown in Figure 12 [51]. Here we just briefly give some typical examples in different research field to elucidate the significance of the dispersion of SWNTs by surfactants. The first example comes from the preparation of advanced functional materials using SDS-coated SWNTs. When subjected to shear flow in a polymer solution, the tubes will be recondensed and 
aligned and finally a nanotube fiber can be obtained which has a high elastic modulus [16]. The surfactant-coated SWNTs can be also used as starting materials for SWNTs sorting i.e., separating semiconducting tubes from metallic ones. In recent years, great progress has been made in this direction by density-gradient ultracentrifugation and tubes with defined diameter and chirality can be obtained (Figure 13) [52,53]. The sorting of SWNTs is necessary and important especially in the fabrication of high performance nanodevices such as field effect transistor arrays [54].
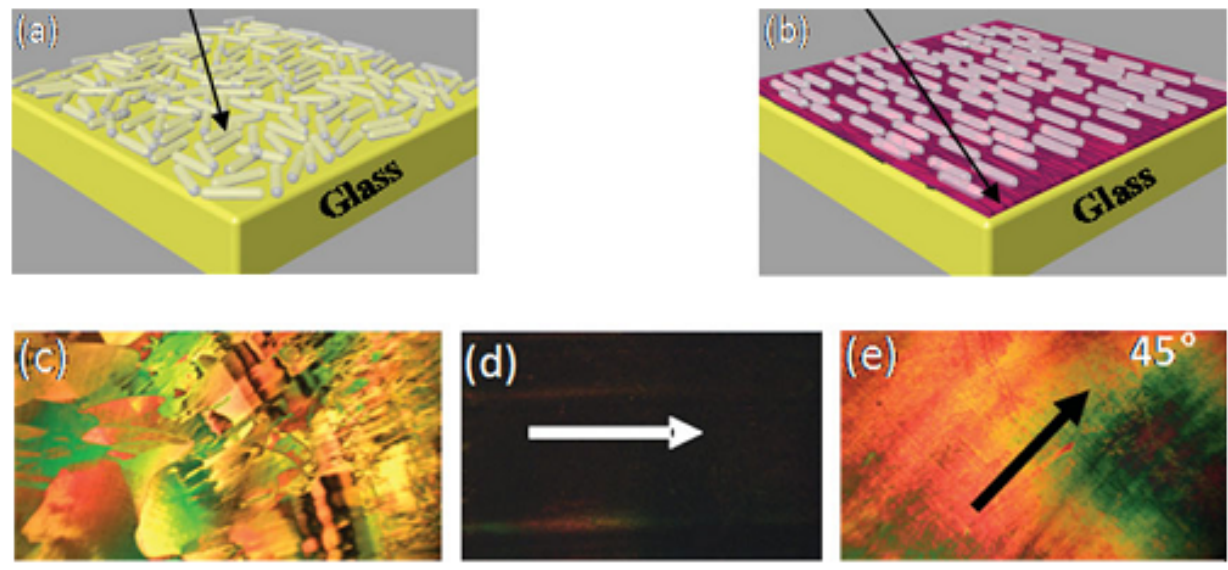

Figure 12. The aligned SWNT/polymer films can be used to orient the molecules of the liquid crystal. a) Schematic diagrams for arrays of LCs on a glass substrate showing random directional local phases. b) On a shear-aligned composite thin film (1a-SWNTarc-discharge 1:1, $2 \mathrm{mg} \mathrm{mL}^{-1}$ in DCB) showing uniformly aligned LC domains. c) Transmission cross-polarized microscope image of a drop of LC 5CB on a bare glass substrate showing many small randomly aligned domains. d) A drop of LC 5CB on a glass substrate with sheared composite thin filmwith the shearing direction parallel to one of the polarizers. e) Shearing direction $45^{\circ}$ to one of the polarizers. Arrows indicate the shearing direction [51].

Due to the unique geometry, ideal size and low cytotoxicity, SWNTs have great potential applications in life science. For this purpose the surfactant used to disperse SWNTs should be biocompatible and nonimmunogenity. One good choice is lipid derivative with a PEO segment as the hydrophilic part $[55,56]$. SWNTs functionalized with single stranded-DNA can find application in gene therapy since the tubes can transport the cell membranes [55]. SWNTs are also known to absorb the near infrared light which will subsequent induce a local temperature rise, as shown in Figure 14. The surfactant-coated SWNTs can thus be used in photothermal treatments in some diseases such as cancer [55]. Besides, other functionalities including fluorescent dyes, cell receptors and drugs can be also integrated onto the surfactant-coated SWNTs to construct multifunctional materials. 
$\mathbf{a}$
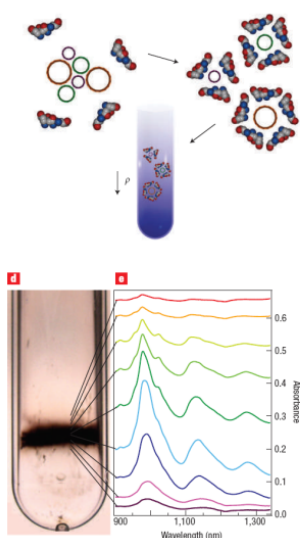

Б
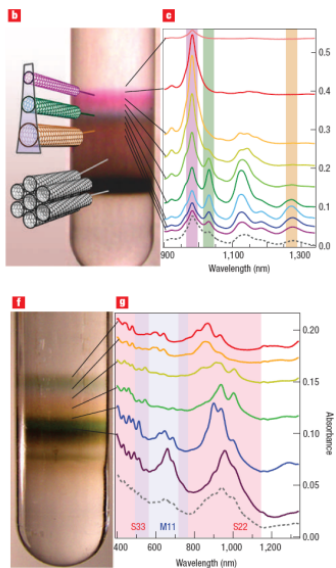

Figure 13. Sorting of SWNTs by diameter, bandgap and electronic type using density gradient ultracentrifugation. a, Schematic of surfactant encapsulation and sorting, where $r$ is density. B-g, Photographs and optical absorbance $(1 \mathrm{~cm}$ path length) spectra after separation using density gradient ultracentrifugation. A rich structure-density relationship is observed for SC-encapsulated SWNTs, enabling their separation by diameter, bandgap and electronic type. In contrast, no separation is observed for SDBS-encapsulated SWNTs. b,c, SC encapsulated, CoMoCAT-grown SWNTs (7-11 $\left.A^{\circ}\right)$. d, e, SDBS-encapsulated CoMoCAT-grown SWNTs (7-11 $\left.A^{\circ}\right)$. f, g, SC-encapsulated, laser-ablation-grown SWNTs (11-16 $\left.\mathrm{A}^{\circ}\right) \cdot \mathrm{pH}=7$ for all parts. SWNTs before sorting are depicted as a dashed grey line in $\mathbf{c}$ and $\mathbf{g}$ [53].
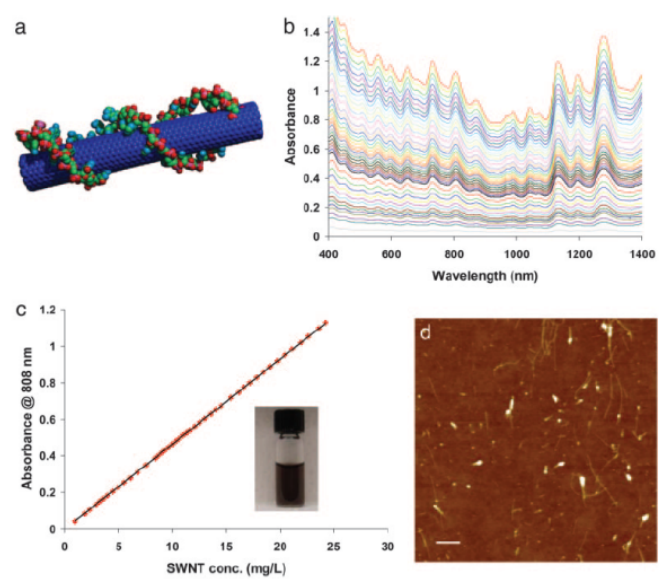

Figure 14. Carbon nanotubes with high NIR absorbance solubilized in water. (a) Schematic of a Cy3-DNA-functionalized SWNT. The drawing is only a graphic presentation and does not represent the precise way DNA binds on SWNTs. (b) UV-visible spectra of solutions of individual SWNTs functionalized noncovalently by 15-mer Cy3 labeled-DNA at various nanotube concentrations. (c) Absorbance at $808 \mathrm{nmvs}$.SWNTconcentration (optical path $=1 \mathrm{~cm}$ ). Solid line is Beer's law fit to obtain molar extinction coefficient of SWNT $7.9 \times 10^{6} \mathrm{M}^{-1} \bullet \mathrm{cm}^{-1}$. (Inset) A photo of a DNA functionalized SWNT solution. (d) AFM image of DNA-functionalized individual SWNTs (height of 1-10 nm) deposited on a SiO2 substrate (Scale bar: $200 \mathrm{~nm}$.) [55]. 


\section{Summary and outlook}

The combined development of colloid and interface science and nanotechnology has paved an effective way to disperse CNTs, especially SWNTs, into water using surfactants. Up to now, almost all the common surfactants have been tested and quite a few newly synthesized surfactants with unique molecular structures have been tried. The effective dispersing methodology has been found and a general dispersing mechanism has been proposed. These advances enable us to get SWNTs dispersions up to single tube level, which significantly facilitates the property manipulation of the tubes and leads to a variety of important applications of SWNTs in physics, biology and life science.

At the same time, one should also keep in mind that challenges still exist. Although good dispersions with large amount of tubes or single tubes can be obtained individually, a combination of them, i.e., dispersions with large amount of single tubes, is still difficult to get. Achievement at this point will rely on the appearance of novel surfactant with improved performance and/or further optimization of the dispersion methodology. In nanodevice fabrication, the insulating surfactant layer on the tube surface may be undesirable and effective removal of them might be an issue. Despite these limitations, dispersing SWNTs using surfactants has provide an elegant way to manipulate these interesting nanomaterials and we believe the challenges mentioned above will be conquered in near future based on the continuous efforts made by scientists from related disciplines.

\section{Acknowledgments}

The authors gratefully acknowledge support of this work by the Natural Science Foundation of China (21203109) and (20873077) and the program of Hundreds of Talents of the Chinese Academy of Sciences.

\section{Author details}

Xia Xin ${ }^{1}$, Guiying $\mathrm{Xu}^{1}$ and Hongguang $\mathrm{Li}^{2}$

1 National Engineering Technology Research Center for Colloidal Materials, Shandong University, Jinan, , P. R. China

2 Laboratory of Clean Energy Chemistry and Materials, Lanzhou Institute of Chemical Physics, Chinese Academy of Sciences, Lanzhou, , P. R. China 


\section{References}

[1] Kong J, Franklin NR, Zhou C, Chapline MG, Peng S, Cho K, Dai H. Nanotube molecular wires as chemical sensors. Science 2000; 287: 622-5.

[2] Tasis D, Tagmatarchis N, Bianco A, Prato M. Chemistry of carbon nanotubes. Chem. Rev 2006; 106: 1105-36.

[3] Karousis N, Tagmatarchis N. Current progress on the chemical modification of carbon nanotubes. Chem. Rev. 2010; 110: 5366-97.

[4] Ball P. Roll up for the revolution. Nature 2001; 414: 142-4.

[5] Iijima S. Helical microtubes of graphitic cabon. Nature 1991; 354: 56-8.

[6] Bachilo, S. M., Strano, M. S., Kittrell. C., Hauge, R. H., Smalley, R. E., \& Weisman, R. B. Structure-assigned optical spectra of single-walled carbon nanotubes. Science (2002). , 298, 2361-6.

[7] Wu J, Gerstandt K, Zhang H, Liu J, Hinds B. Electrophoretically induced aqueous flow through single-walled carbon nanotube membranes. Nature Nanotechno. 2012; 7 : 133-9.

[8] Zhou W, Ding L, Yang S, Liu J. Synthesis of high-density, large-diameter, and aligned single-walled carbon nanotubes by multiple-cycle growth methods. ACS Nano 2011; 5: 3849-57.

[9] Strano, M. S., Dyke, C. A., Usrey, M. L., Barone, P. W., Allen, M. J., Shan. H., Kittrell, C., Hauge, R. H., Tour, J. M., \& Smalley, R. E. Electronic structure control of singlewalled carbon nanotube functionalization. Science (2003)., 301, 1519-22.

[10] Vaisman L, Wagner HD, Marom G. The role of surfactants in dispersion of carbon nanotubes, Advances in Colloid and Interface Science 2006; 128-130: 37-46.

[11] Wang H, Dispersing carbon nanotubes using surfactants. Curr. Opin. Colloid Interface Sci. 2009; 14: 364-71.

[12] Taylor DJF, Thomas RK, Penfold J. Polymer/surfactant interactions at the air/water interface, Adv. Colloid and Interface Sci. 2007; 132: 69-110.

[13] Wenseleers, W., Vlasov, I. I., Gppvaerts, E., Obraztsova, E. D., Lobach, A. S., \& Bouwen. A. Efficient isolation and solubilization of pristine single-walled nanotubes in bile salt micelles. Adv. Funct. Mater. (2004)., 14, 1105-12.

[14] Strano, M. S., Moore. V. C., Miller, M. K., Allen, M. J., Haroz. E. H., Kittrell, C., Hauge, R. H., \& Smalley, R. E. The role of surfactant adsorption during ultrasonication in the dispersion of single-walled carbon nanotubes. J Nanosci Nanotech (2003)., $3,81-6$.

[15] O'Connell, M. J., Bachilo. S. M., Huffman, C. B., Moore, V. C., Strano, Haroz. E. H., Rialon, K. L., Boul, P. J., Noon, W. H., Kittrell, C., Ma, J., Hauge, R. H., Weisman,R. 
B., \& Smalley, R. E. Band gap fluorescence from individual single-walled carbon nanotubes. Science (2002)., 297, 593-6.

[16] Pang JY, Xu GY, Tan YB, He F. Water-dispersible carbon nanotubes from a mixture of an ethoxy-modified trisiloxane and pluronic block copolymer F127. Colloid Polym. Sci. 2010; 288: 1665-75.

[17] Pang JY, Xu GY, Yuan SL, Tan YB, He F. Dispersing carbon nanotubes in aqueous solutions by a silicon surfactant: Experimental and molecular dynamics simulation study. Colloids Surf. A 2009; 350: 101-8.

[18] Panagiotis A, Andrei G, Ailsa L, Nerushev O, Bock H, Campbell EEB. Dispersing individual single-wall carbon nanotubes in aqueous surfactant solutions below the cmc. J. Phys. Chem. B 2010; 114: 2-9.

[19] Alvarez NT, Kittrell C, Schmidt HK, Hauge RH, Engel PS, Tour JM. Selective photochemical functionalization of surfactant-dispersed single wall carbon nanotubes in water. J. Am. Chem. Soc. 2008; 130: 14227-33.

[20] Moore, V. C., Strano, M. S., Haroz, E. H., Hauge, R.H., \& Smalley, R. E. Individually suspended single-walled carbon nanotubes in various surfactants. Nano Lett. (2003)., 3, 1379-82.

[21] Nativ-Roth E, Yerushalmi-Rozen R, Regev O. Phase behavior and shear alignment in SWNT-surfactant dispersions. Small 2008; 4: 1459-67.

[22] Döle, S., Lechner, B. D., Park. J. H., Schymura, S., Lagerwall, J. P. F., \& Scalia, G. Utilizing the krafft phenomenon to generate ideal micelle-free surfactant-stabilized nanoparticle suspensions. Angew. Chem. Int. Ed. (2012)., 51, 3254-57.

[23] Gong HJ, Xu GY, Liu T, Pang JY, Dou WL, Xin X. Synthesis of block polyethers with various structures and their application in dispersing single-walled carbon nanotubes. Colloid Polym. Sci. 2011; 289: 933-42.

[24] Shvartzman-Cohen R, Nativ-Roth E, Baskaran E, Levi-Kalisman Y, Szleifer I, Yerushalmi-Rozen R. Selective dispersion of single-walled carbon nanotubes in the presence of polymers: the role of molecular and colloidal length scales. J. Am. Chem. Soc. 2004; 126: $14850-7$.

[25] Shvartzman-Cohen R, Levi-Kalisman Y, Nativ-Roth E, Yerushalmi-Rozen R. Generic approach for dispersing single-walled carbon nanotubes: The strength of a weak interaction. Langmuir 2004; 20: 6085-8.

[26] Xin X, Xu GY, Zhang ZQ, Chen YJ, Wang F. Aggregation behavior of star-like PEOPPO-PEO block copolymer in aqueous solution. Eur. Polym. J. 2007; 43: 3106.

[27] Xin X, Xu G, Zhao T, Zhu Y, Shi X, Gong H, Zhang Z. Dispersing carbon nanotubes in aqueous solutions by a star-like block copolymer. J. Phys. Chem. C 2008; 112: 16377. 
[28] Islam MF, Rojas E, Bergey DM, Johnson AT, Yodh AG. High weight fraction surfactant solubilization of single-wall carbon nanotubes in Water. Nano Lett. 2003; 3: 269-73.

[29] Zhou W, Islam MF, Wang H, Ho DL, Yodh AG, Winey KI, et al. Small angle neutron scattering from single-wall carbon nanotube suspensions: evidence for isolated rigid rods and rod networks. Chem. Phys. Lett. 2004; 384: 185-9.

[30] Chen RJ, Zhang YG, Wang DW, Dai HJ. Noncovalent sidewall functionalization of single-walled carbon nanotubes for protein immobilization. J. Am. Chem. Soc. 2001; 123: 3838-9.

[31] Backes C, Schmidt CD, Hauke F, Böttcher C, Hirsch A. High population of individualized SWCNTs through the adsorption of water-soluble perylenes. J. Am. Chem. Soc. 2009; 131: 2172-84.

[32] Takaguchi Y, Tamura M, Sako Y, Yanagimoto Y, Tsuboi S, Uchida T, Shimamura K, Kimura SI, Wakahara T, Maeda Y, Akasaka T. Fullerodendron-assisted dispersion of single-walled carbon nanotubes via noncovalent functionalization. Chem. Lett. 2005; 34: 1608-9.

[33] Li HG, Liu CC, Hao JC, Hartnagel U, Hirsch A. Dispersing carbon nanotubes by starlike water soluble $\mathrm{C}_{60}$ derivatives. J. Nanosci. Nanotechno. 2009; 9: 2763-7.

[34] Grunlan JC, Liu L, Kim YS. Tunable single-walled carbon nanotube microstructure in the liquid and solid states using poly(acrylic acid). Nano Lett. 2006; 6: 911-5.

[35] Bandyopadhyaya R, Nativ-Roth E, Regev O, Yerushalmi-Rozen R. Stabilization of individual carbon nanotubes in aqueous solutions. Nano Lett. 2002; 2: 25-8.

[36] Moulton SE, Maugey M, Poulin P, Wallace GG. Liquid crystal behavior of single-walled carbon nanotubes dispersed in biological hyaluronic acid solutions. J. Am. Chem. Soc. 2007; 129: 9452-57.

[37] Star A, Steuerman DW, Heath JR, Stoddart JF. Starched carbon nanotubes. Angew. Chem. Int. Ed.2002; 41: 2508-12.

[38] Horn DW, Tracy K, Easley CJ, Davis VA. Lysozyme dispersed single-walled carbon nanotubes: Interaction and activity. J. Phys. Chem. C 2012; 116: 10341-8.

[39] Cathcart H, Quinn S, Nicolosi V, Kelly JM, Blau WJ, Coleman JN. Spontaneous debundling of single-walled carbon nanotubes in DNA-based dispersions. J. Phys. Chem. C 2007; 111: 66-74.

[40] Tardani F, Mesa CL, Poulin P, Maugey M. Phase behavior of DNA-based dispersions containing carbon nanotubes: Effects of added polymers and ionic strength on excluded volume. J. Phys. Chem. C 2012; 116: 9888-94.

[41] Yurekli, K., \& Mitchell. C. A., Krishnamoorti. R. Small-angle neutron scattering from surfactant-assisted aqueous dispersions of carbon nanotubes. J. Am. Chem. Soc. (2004)., 126, 9902-3. 
[42] Tummala NR, Striolo A. SDS surfactants on carbon nanotubes: aggregate morphology. ACS Nano 2009; 3: 595-602.

[43] Wallace EJ, Sansom MSP. Carbon nanotube/detergent interactions via coarse-grained molecular dynamics. Nano. Lett. 2007; 7:1923-8.

[44] Shvartzman-Cohen R, Nativ-Roth E, Baskaran E, Levi-Kalisman Y, Szleifer I, Yerushalmi-Rozen R. Selective dispersion of single-walled carbon nanotubes in the presence of polymers: the role of molecular and colloidal length scales. J. Am. Chem. Soc. 2004; 126: 14850-7.

[45] Lynch MD, Patrick DL. Organizing carbon nanotubes with liquid crystals. Nano Lett. 2002; 2: 1197-201.

[46] Lagerwall JPF, Scalia G, Haluska M, Dettlaff-Weglikowska U, Roth S, Giesselmann F. Nanotube alignment using lyotropic liquid crystals. Adv. Mater. 2007; 19: 359-64.

[47] Xin X, Li HG, Wieczorek SA, Szymborski T, Kalwarczyk E, Ziebacz N. Gorecka E, Pociecha D, Hołyst, R. Incorporation of carbon nanotubes into lyotropic liquid crystal by phase separation in the presence of hydrophilic polymer. Langmuir 2010; 26: 3562-8.

[48] Xin X, Li HG, Kalwarczyk E, Kelm A, Fialkowski M, Gorecka E. Pociecha D, Hołyst, R. Single-walled carbon nanotube/lyotropic liquid crystal hybrid materials fabricated by a phase separation method in the presence of polyelectrolyte. Langmuir 2010; 26: 8821-8.

[49] Camponeschi E, Florkowski B, Vance R, Garrett G, Garmestani H, Tannenbaum R. Uniform directional alignment of single-walled carbon nanotubes in viscous polymer flow. Langmuir 2006; 22: 1858-62.

[50] Nativ-Roth E, Regev O, Yerushalmi-Rozen R. Shear-induced ordering of micellar arrays in the presence of single-walled carbon nanotubes. Chem. Commun. 2008; 17: 2037-9.

[51] Lee HW, You W, Barman S, Hellstrom S, LeMieux MC, Oh JH, Liu SH, Fujiwara T, Wang WM, Chen B, Jin YW, Kim JM, Bao ZN. Lyotropic liquid-crystalline solutions of high-concentration dispersions of single-walled carbon nanotubes with conjugated polymers. Small 2009; 5: 1019-24.

[52] Ghosh S, Bachilo SM, Weisman RB. Advanced sorting of single-walled carbon nanotubes by nonlinear density-gradient ultracentrifugation. Nature Nanotechno. 2010; 5: 443-50.

[53] Arnold MS, Green AA, Hulvat JF, Stupp SI, Hersam MC. Sorting carbon nanotubes by electronic structure using density differentiation. Nature Nanotechno. 2006; 1: 60-5.

[54] LeMieux MC, Roberts M, Barman S, Jin YW, Kim JM, Bao ZN. Self-sorted, aligned nanotube networks for thin-film transistors. Science 2008; 321: 101-4. 
[55] Kam NWS, O'Connell M, Wisdom JA, Dai HJ. Carbon nanotubes as multifunctional biological transporters and near-infrared agents for selective cancer cell destruction. PNAS 2005; 102: 11600-5.

[56] Liu Z, Davis C, Cai WB, He LN, Chen XY, Dai HJ. Circulation and long-term fate of functionalized, biocompatible single-walled carbon nanotubes in mice probed by Raman spectroscopy. PNAS 2008; 105: 1410-5. 
